\title{
Anisotropic but Nodeless Superconducting Gap in the Presence of Spin-Density Wave in Iron-Pnictide Superconductor $\mathrm{NaFe}_{1-x} \mathrm{Co}_{x} \mathrm{As}$
}

\author{
Q. Q. Ge (葛青亲), ${ }^{1}$ Z. R. Ye (叶子荣), ${ }^{1}$ M. Xu (徐敏), ${ }^{1}$ Y. Zhang (张炎), ${ }^{1}$ J. Jiang (姜娟), ${ }^{1}$ B. P. Xie (谢斌平), ${ }^{1}$ \\ Y. Song (宋宇), ${ }^{2}$ C. L. Zhang (张承林), ${ }^{2}$ Pengcheng Dai (戴鹏程), ${ }^{2,3}$ and D. L. Feng (封东来) $)^{1, *}$ \\ ${ }^{1}$ State Key Laboratory of Surface Physics, Department of Physics, and Advanced Materials Laboratory, \\ Fudan University, Shanghai 200433, People's Republic of China \\ ${ }^{2}$ Department of Physics and Astronomy, The University of Tennessee, Knoxville, Tennessee 37996-1200, USA \\ ${ }^{3}$ Beijing National Laboratory for Condensed Matter Physics, Institute of Physics,
}

Chinese Academy of Sciences, Beijing 100190, China

(Received 10 September 2012; published 18 March 2013)

\begin{abstract}
The coexisting regime of spin-density wave (SDW) and superconductivity in iron pnictides represents a novel ground state. We have performed high-resolution angle-resolved photoemission measurements on $\mathrm{NaFe}_{1-x} \mathrm{Co}_{x} \mathrm{As}(x=0.0175)$ in this regime and revealed its distinctive electronic structure, which provides some microscopic understandings of its behavior. The SDW signature and the superconducting gap are observed on the same bands, illustrating the intrinsic nature of the coexistence. However, because the SDW and superconductivity are manifested in different parts of the band structure, their competition is nonexclusive. Particularly, we find that the gap distribution is anisotropic and nodeless, in contrast to the isotropic superconducting gap observed in a SDW-free $\mathrm{NaFe}_{1-x} \mathrm{Co}_{x} \mathrm{As}(x=0.045)$, which puts strong constraints on theory.
\end{abstract}

Subject Areas: Condensed Matter Physics, Superconductivity

Most unconventional superconductors appear in the vicinity of a certain magnetically ordered phase [1]. It is suggested that magnetism plays a critical role in the pairing mechanisms of the cuprates [2], heavy Fermion superconductors [2,3], and even organic superconductors [4]. For iron-pnictide superconductors, a spin-density wave (SDW) phase appears next to the superconducting (SC) phase [5-7], and, in some cases, they even coexist [8-13], which gives a unique $\mathrm{SC}$ ground state. While the coexisting SDW and SC phases may have a significant impact on the SC mechanism [9], much is not clear about the subtle interacting nature between magnetism and superconductivity [14]. In fact, theories based on $s^{++}$pairing symmetry suggest that there must be nodes in the SC gap in this regime [15], and the coexisting SDW and SC phases cannot be microscopic [9]. On the other hand, theories based on $s^{+-}$pairing symmetry suggest nodeless SC gaps in the presence of weak magnetic order; moreover, the coexistence may cause angular variation of the SC gap and even give rise to nodes in the limit of strong antiferromagnetic (AFM) ordering $[15,16]$, as indicated in a thermal conductivity study on $\mathrm{Ba}_{1-x} \mathrm{~K}_{x} \mathrm{Fe}_{2} \mathrm{As}_{2}$ [17].

The coexistence of SDW and superconductivity in various iron pnictides has been illustrated by neutron scattering [8-12], nuclear magnetic resonance [18,19], and angle-

\footnotetext{
*dlfeng@fudan.edu.cn
}

Published by the American Physical Society under the terms of the Creative Commons Attribution 3.0 License. Further distribution of this work must maintain attribution to the author(s) and the published article's title, journal citation, and DOI. resolved photoemission spectroscopy (ARPES) experiments [13]. Recent scanning tunneling microscope (STM) studies show the real-space coexistence and competition of SDW and superconductivity in $\mathrm{NaFe}_{1-x} \mathrm{Co}_{x} \mathrm{As}$ $[20,21]$. However, so far, little is known regarding the electronic structure of the coexisting phase in the momentum space, such as its SC gap distribution, and how the two orders coexist and compete in the same electronic structure. In this paper, we report ARPES studies on $\mathrm{NaFe}_{0.9825} \mathrm{Co}_{0.0175} \mathrm{As}$ in this coexisting regime. The bandstructure reconstruction corresponding to the SDW formation and the SC gap could be observed on the same bands, which provides direct evidence for the intrinsic coexistence of the two orders. We find that SDW formation does not cause much depletion of the states near the Fermi energy $\left(E_{F}\right)$; therefore, this formation allows the superconductivity to occur. Moreover, the SC gap distribution is found to be nodeless on all Fermi surface sheets: It is isotropic on the hole pocket, but it is highly anisotropic on the electron pockets. Our results reveal the distinct electronic properties of the coexisting phase and provide explicit constraints on theory.

High-quality $\mathrm{NaFe}_{0.9825} \mathrm{Co}_{0.0175}$ As single crystals were synthesized by the self-flux method described elsewhere [22]. The $\mathrm{SC}$ transition temperature $\left(T_{c}\right)$ is determined by the magnetic susceptibility measurements with a SQUID magnetometer [Fig. 1(a)], which shows an onset drop at 20.5 K. Resistivity measured by a physical properties measurement system (PPMS, Quantum Design, Inc.) indicates zero resistivity below $18 \mathrm{~K}$ and a structural transition at $T_{S}=36 \mathrm{~K}$. Our neutron scattering data show that the SDW transition temperature $\left(T_{N}\right)$ is $28 \mathrm{~K}$ [Fig. 1(b)]. 
(a)

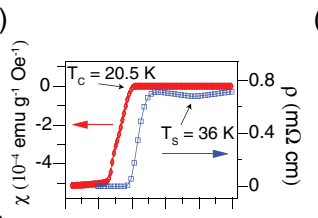

(b)

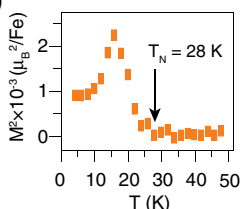

(c)

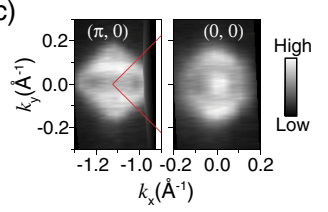

(d)
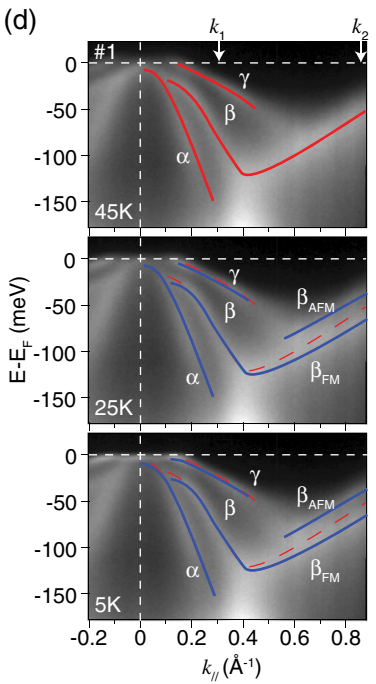

$k_{2}(\mathrm{e})$

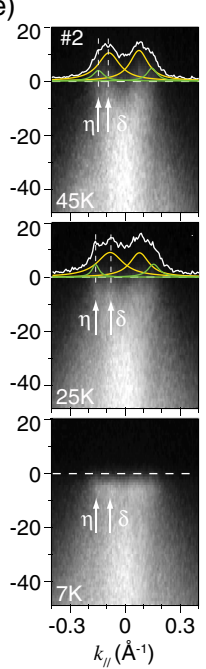

(f)
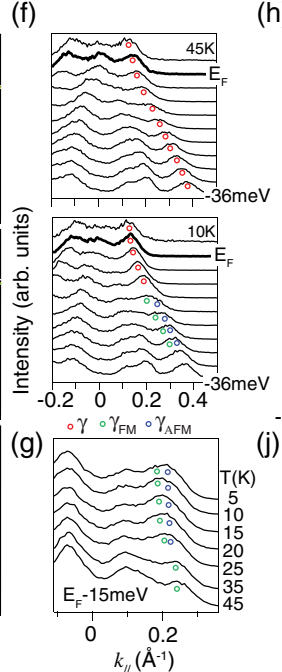

(h)
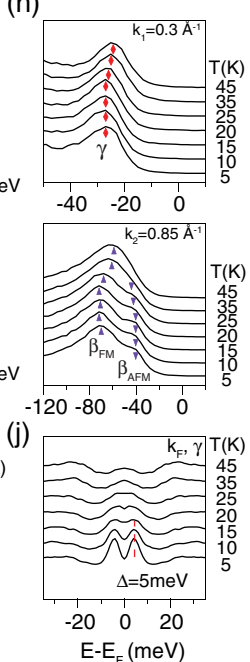

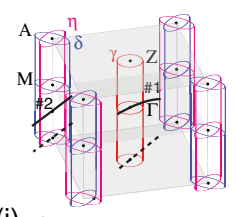

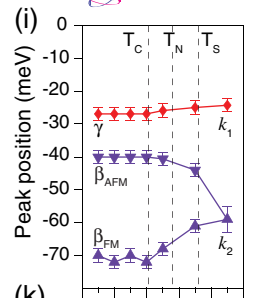

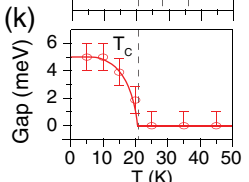

FIG. 1. (a) Magnetic susceptibility of a $\mathrm{NaFe}_{0.9825} \mathrm{Co}_{0.0175} \mathrm{As}$ single crystal taken at a magnetic field of 20 Oe in the zero-field cool mode, and its resistivity as a function of temperature. (b) Temperature dependence of the magnetic-order parameter at $Q=(1,0,1.5)$ for $\mathrm{NaFe}_{0.9825} \mathrm{Co}_{0.0175} \mathrm{As}$ measured by neutron scattering. (c) Photoemission intensity map at the Fermi energy integrated over $\left[E_{F}-5 \mathrm{meV}, E_{F}+5 \mathrm{meV}\right]$. (d) The band structure of $\mathrm{NaFe}_{0.9825} \mathrm{Co}_{0.0175} \mathrm{As}$ at 45,25 , and $5 \mathrm{~K}$, respectively, along cut \#1 across $\Gamma$, as indicated in the inset. The dashed lines in the lower panels are the band dispersion at $45 \mathrm{~K}$, for comparison purposes. (e) Temperature dependence of the band structure around the zone corner along cut \#2, as indicated in the inset. The MDCs (momentum-distribution curves) at $E_{F}$ are plotted on the 25 and $45 \mathrm{~K}$ data. Each MDC was fitted to four Lorentzians (overlaid yellow and green lines). (f) MDCs near the zone center at 45 and $10 \mathrm{~K}$. (g) Temperature dependence of the MDC at $E_{F}-15$ meV near the zone center. The positions of the $\gamma$ band are marked in panels (f) and (g). (h) Temperature dependence of the EDCs (energy-distribution curves) at selected momenta: $k_{1}=0.3 \AA^{-1}$ and $k_{2}=0.85 \AA^{-1}$, respectively, as marked in panel (d). Because of the broad line shape, $\gamma_{\mathrm{AFM}}$ and $\gamma_{\mathrm{FM}}$ are not resolved, but the shift of the overall features is obvious. (i) The temperature dependence of the peak positions in panel (h). (j) The temperature dependence of the symmetrized EDCs measured at the $k_{F}$ of $\gamma$. (k) The temperature dependence of the SC gap of $\gamma$. The gap size is estimated through an empirical fit, as described in detail in Ref. [36]. The inset on the top-right corner shows the Fermi surface of $\mathrm{NaFe}_{0.9825} \mathrm{Co}_{0.0175}$ As. The two solid lines mark cut \#1 and cut \#2, along which the data in panels (d) and (e) are located, respectively. The two dashed lines on the bottom plane are their projections. The photoemission data in panels (c) and (e) were acquired in-house, and others were collected at SSRL.

ARPES data were taken with various photon energies in circular polarization at the 1-cubed beam line of BESSY-II; other photoemission measurements were performed either with $21 \mathrm{eV}$ photons at beam line 5-4 of the Stanford Synchrotron Radiation Laboratory (SSRL) or with randomly polarized $21.2 \mathrm{eV}$ light from an in-house SPECS UVLS helium discharging lamp at Fudan University. All the data were taken with SCIENTA R4000 electron analyzers; the overall resolution is set to $6 \mathrm{meV}$ or better, and the typical angular resolution is $0.3^{\circ}$. The samples were cleaved in situ and measured under ultrahigh vacuum, so the aging effects are negligible in the data.

The general electronic structure of $\mathrm{NaFe}_{0.9825} \mathrm{Co}_{0.0175} \mathrm{As}$ is rather similar to the well-studied NaFeAs [22-24]. Figure 1(c) shows the photoemission intensity map near $E_{F}$ taken at $7 \mathrm{~K}$ with $21.2 \mathrm{eV}$ photons. One can observe a hole pocket and a small patchlike feature point around $\Gamma(0,0)$, and two orthogonal elliptical pockets around the zone corner. The photoemission intensity along cut \#1 across $\Gamma$ is plotted in Fig. 1(d), where three bands, $\alpha, \beta$, and $\gamma$, can be resolved, but only $\gamma$ crosses $E_{F}$ and gives the hole Fermi surface. The band top of $\alpha$ is just below $E_{F}$, and it contributes to the small patch in the zone center. Figure 1(e) plots the photoemission intensities at the zone corner, where two electronlike bands, $\delta$ and $\eta$, can be observed. As a previous photon-energy-dependent study has revealed the negligible $k_{z}$ dispersion of NaFeAs [23], the overall Fermi surface topology of $\mathrm{NaFe}_{0.9825} \mathrm{Co}_{0.0175} \mathrm{As}$ is summarized in the inset on the top-right corner of Fig. 1.

The signature of SDW on the electronic structure has been extensively studied before [22,24-26], which is mainly manifested as a remarkable band reconstruction. As shown in Fig. 1(d), $\beta$ shifts significantly with decreased temperature. To illustrate the subtle band reconstruction of $\gamma$, Fig. 1(f) plots the momentum distribution curves (MDCs) near the Fermi crossing of $\gamma$ at several binding energies near $E_{F}$ at 45 and $10 \mathrm{~K}$, and Fig. $1(\mathrm{~g})$ plots the MDC at $E_{F}-15 \mathrm{meV}$ as a function of temperature. It is clear that $\gamma$ first shifts in one direction because of the SDW [24] and then splits into two at low temperatures. Our recent ARPES study on the mechanically detwinned $\mathrm{NaFeAs}$ has shown that the $\beta$ and $\gamma$ bands disperse differently along the ferromagnetic (FM) and AFM directions, which gives the appearance of band splitting in the twinned 
sample here, as noted by the subscripts in Fig. 1 [24]. Similar reconstruction effects can be observed in the energy distribution curves (EDCs), as well, in Fig. 1(h). As shown by the temperature dependence of the EDC peak positions summarized in Fig. 1(i), the electronic structure reconstruction occurs above the structural transition because of the fluctuations of the SDW and electronic structure nematicity [24,27]. This reconstruction evolves smoothly across the structural and Neel transitions and saturates below $20 \mathrm{~K}$, with the separation of $\beta_{\mathrm{AFM}}$ and $\beta_{\mathrm{FM}}$ reaching $32 \mathrm{meV}$ and the shift of $\gamma$ reaching $3 \mathrm{meV}$. The reconstruction of $\delta$ and $\eta$ is subtle; nevertheless, in Fig. 1(e), their features in the MDCs at $E_{F}$ clearly show finite shifts as well [24]. On the other hand, the SC gap opens just below $T_{c}$, as illustrated by the symmetrized EDCs of the $\gamma$ band with respect to $E_{F}$ in Fig. 1(j) and the fitted SC gap in Fig. 1(k). The fact that the signatures of both the superconductivity and SDW emerge in the same band structure confirms their intrinsic coexistence. Furthermore, the band reconstruction from SDW mainly occurs over large energy and momentum scales for $\beta$ below $E_{F}$, and it leaves the states on all the Fermi surfaces largely intact in this doping regime. Therefore, superconductivity could occur in the presence of SDW in this regime.

The SC gap is mapped out extensively over the entire Brillouin zone. Figure 2(a) shows the symmetrized photoemission intensity along four momentum cuts across the $\gamma$ (a)

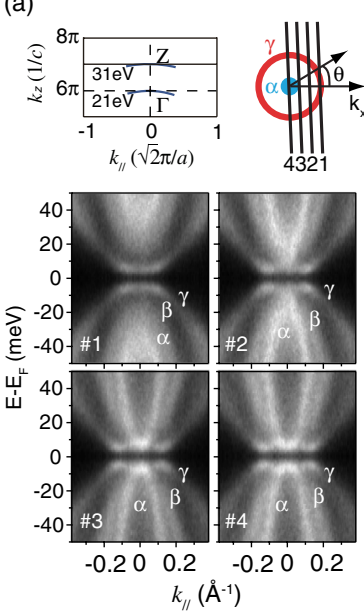

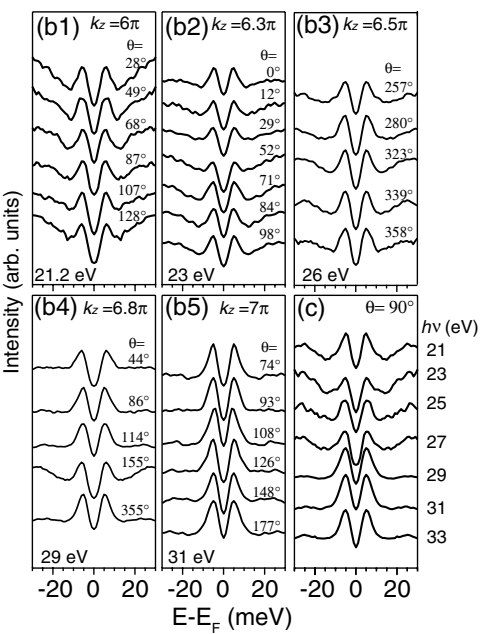

FIG. 2. (a) The photoemission intensities taken with $21.2 \mathrm{eV}$ photons at $7 \mathrm{~K}$ near the zone center, as shown by cuts \#1-\#4 in the inset. The inset to the left shows the momentum cuts sampled by the 21 - and $31-\mathrm{eV}$ photons in the $k_{x}-k_{z}$ cross section of the extended Brillouin zone. (b) The symmetrized spectra at the marked polar angles on the $\gamma$ Fermi surface measured at five typical $k_{z}$ values, with (b1) 21.2-, (b2) 23-, (b3) 26-, (b4) 29-, and (b5) $31-\mathrm{eV}$ photons. (c) $k_{z}$ dependence of the symmetrized spectra measured on the $\gamma$ Fermi surface of another sample at $\theta=90^{\circ}$. The $21.2-\mathrm{eV}$ data were collected at $7 \mathrm{~K}$ with a helium lamp, while the others were collected at $1 \mathrm{~K}$ at BESSY-II. hole Fermi surface in the $k_{z}=6 \pi$ plane. The suppression of the spectral weight around $E_{F}$ indicates the opening of the SC gap. In Fig. 2 (b1), the symmetrized EDCs along the $\gamma$ pocket clearly show sharp coherent peaks and SC gaps of similar amplitude. Data from other $k_{z}$ planes in Figs. 2 (b2)-(b5), and data from another sample taken with more photon energies in Fig. 2(c), show that the gap is isotropically $5 \mathrm{meV}$ on the $\gamma$ pocket, as summarized in Fig. 4(a).

Now we turn to the SC gap on the electron Fermi surfaces around the zone corner. Figure 3(a) shows symmetrized photoemission intensity for six momentum cuts across the $\delta / \eta$ pockets in the $k_{z}=6 \pi$ plane, where the SC gap opens on both Fermi surfaces. Collecting the symmetrized EDCs at various $k_{F}$ 's along the $\delta$ pocket, Fig. 3 (b1) demonstrates an anisotropic gap distribution, where the gap is about $7 \mathrm{meV}$ in the flat part of the ellipse and significantly drops to $4 \mathrm{meV}$ near $\theta=0^{\circ}, 180^{\circ}$. Moreover, such a behavior is observed for all five sampled $k_{z}$ 's, as shown in Figs. 3 (b1)-(b5). Similarly, such an anisotropic gap distribution is observed for $\eta$ but rotated by $90^{\circ}$ [Figs. 3 (c1)-(c5)]. The weak $k_{z}$ dependence is further illustrated with more data taken at $k_{z}=5.5 \pi, 6.3 \pi$, and $6.5 \pi$ with 21,28 , and $30 \mathrm{eV}$ photons, respectively, in Fig. S1 in the Supplemental Material [28].

The gap distribution of $\mathrm{NaFe}_{0.9825} \mathrm{Co}_{0.0175}$ As is summarized in Figs. 4(a)-4(c). The gaps along the $\gamma$ hole Fermi surface show isotropic distribution, while the gaps on the $\delta$ and $\eta$ pockets vary significantly from 4 to $7 \mathrm{meV}$. As a comparison, Figs. 4(d) and 4(e) show the isotropic in-plane gap distribution on individual Fermi surfaces for a SDWfree $\mathrm{NaFe}_{0.955} \mathrm{Co}_{0.045} \mathrm{As}$ sample $\left(T_{c}=20 \mathrm{~K}\right)$, which are retrieved from the symmetrized EDCs provided in Fig. S2 in the Supplemental Material [28]. The gap is about $5 \mathrm{meV}$ on the hole pocket and $5.4 \mathrm{meV}$ on the electron pockets. Such an isotropic in-plane gap distribution has been observed before in $\mathrm{NaFe}_{0.95} \mathrm{Co}_{0.05}$ As as well [29]. Furthermore, Fig. 4(f) compares both the Fermi surfaces and the $\mathrm{SC}$ gap distributions of $\mathrm{NaFe}_{0.9825} \mathrm{Co}_{0.0175} \mathrm{As}$ and $\mathrm{NaFe}_{0.955} \mathrm{Co}_{0.045} \mathrm{As}$. The hole pocket of $\mathrm{NaFe}_{0.955} \mathrm{Co}_{0.045} \mathrm{As}$ is slightly smaller, as expected from cobalt doping, and the ellipticity of its electron pockets is smaller as well.

So far in ARPES experiments, the in-plane anisotropy of the SC gap has been observed only for LiFeAs [30,31], Fe (Te,Se) [32], $\mathrm{KFe}_{2} \mathrm{As}_{2}$ [33], and $\mathrm{Ba}_{1-x} \mathrm{~K}_{x} \mathrm{Fe}_{2} \mathrm{As}_{2}$ [34] among all the iron-based superconductors, but none of them is in the coexisting regime. The small gap anisotropy reported on one of the hole pockets of $\mathrm{Ba}_{1-x} \mathrm{~K}_{x} \mathrm{Fe}_{2} \mathrm{As}_{2}$ is within the experimental error, considering less than $0.6 \mathrm{meV}$ difference over the $9-10 \mathrm{meV}$ gap amplitude [34]. The moderately anisotropic gap on a hole Fermi surface of LiFeAs might be a mere consequence of the Fermi surface topology, since it is qualitatively consistent with the gap function $\triangle(k)=\triangle_{0} \cos k_{x} \cos k_{y}$ predicted based on the $s^{+-}$pairing symmetry [30,31]. For 

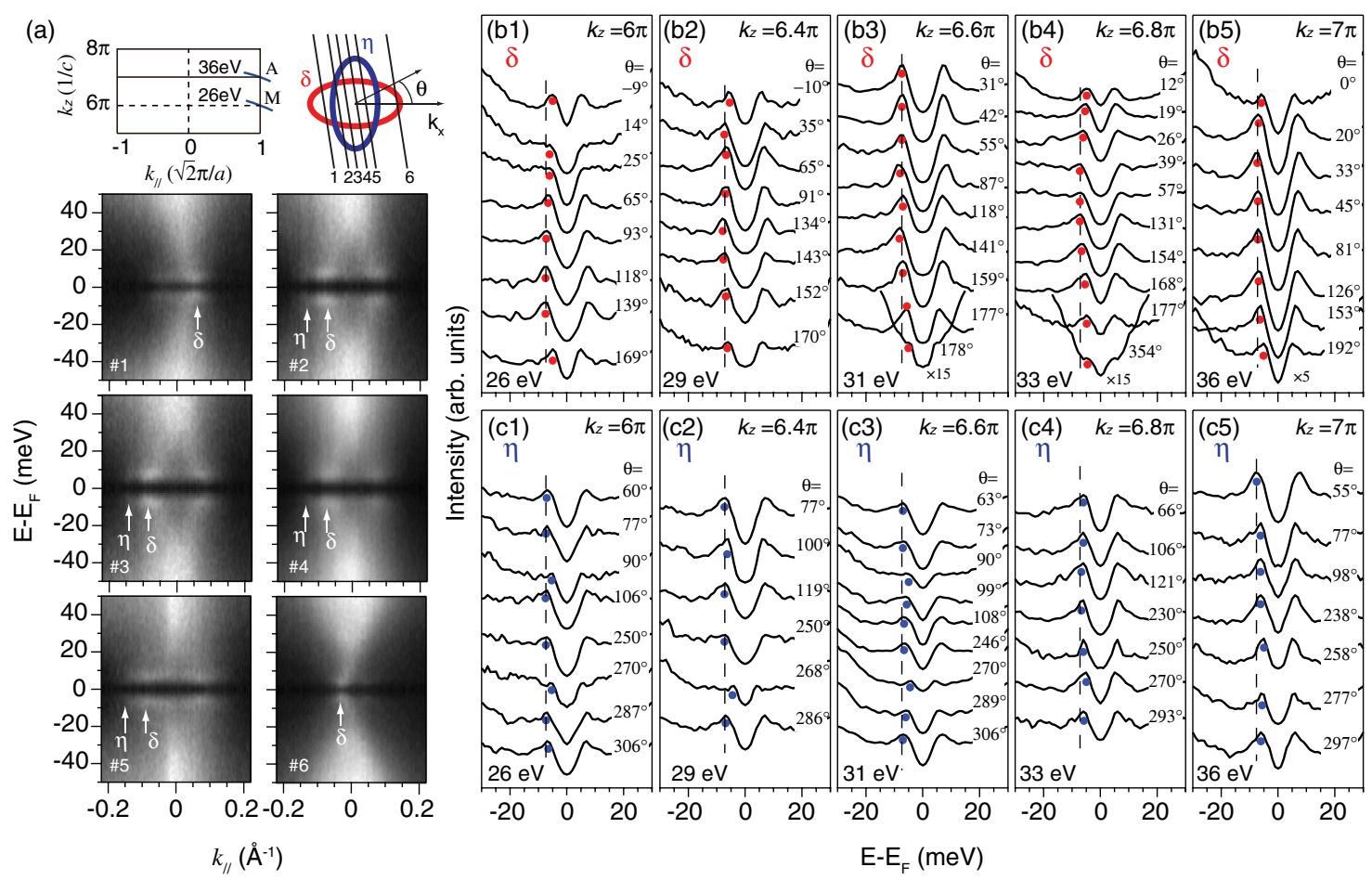

FIG. 3. (a) The photoemission intensities taken with $26 \mathrm{eV}$ photons at $1 \mathrm{~K}$ near the zone corner, as shown by cuts \#1-\#6 in the topright inset. The top-left inset shows the momentum cuts sampled by the 26- and 36-eV photons in the $k_{x}-k_{z}$ cross section of the Brillouin zone. (b) The symmetrized spectra at the marked polar angles on the $\delta$ Fermi surface measured at five typical $k_{z}$ 's with (b1) 26-, (b2) 29-, (b3) 31-, (b4) 33-, and (b5) 36-eV photons. The dashed lines here are guides to the eye for the variation of the SC gaps. (c1)-(c5) Same as in panels (b1)-(b5), but on the $\eta$ electron Fermi surface. All data were collected at 1 K at BESSY-II. Note that the bottom curves in panels (b3), (b4), and (b5) are magnified vertically.

$\mathrm{NaFe}_{0.9825} \mathrm{Co}_{0.0175} \mathrm{As}$, the large ellipticity gives a variation of $\left|\cos k_{x} \cos k_{y}\right|$ from around 0.98 in the flat region to around 0.91 on the tip, which could not explain the over $40 \%$ change of the gap based on the Fermi surface topology. We note that an anisotropic gap distribution around the zone corner has also been revealed in LiFeAs, which deviates from the canonical $s^{+-}$-wave gap function and is explained in terms of the band hybridization [31]. Consistently, the deviation is most prominent around $\theta=$ $45^{\circ}$, where the hybridization is the strongest. However, the anisotropic behavior in $\mathrm{NaFe}_{0.9825} \mathrm{Co}_{0.0175}$ As deviates from the gap function remarkably around $\theta=0^{\circ}$ and $90^{\circ}$, which is away from the Fermi surface region of mixed orbital character. For $\mathrm{Fe}(\mathrm{Te}, \mathrm{Se})$, the anisotropy of the SC gap on the hole pocket is suggested to be a consequence of sizable second-nearest-neighbor interactions, while the anisotropic and nodal gaps on a hole pocket of $\mathrm{KFe}_{2} \mathrm{As}_{2}$ may be related to strong intrapocket scattering [35], or specific orbital characters near $Z$ [36]. Alternatively, the angular variation in the $d_{x y}$ orbital content of the $\gamma$ Fermi surface was predicted to cause an anisotropic gap distribution on the electron pockets [35]. However, since $\mathrm{NaFe}_{0.9825} \mathrm{Co}_{0.0175} \mathrm{As}$ and $\mathrm{NaFe}_{0.955} \mathrm{Co}_{0.045}$ As have similar Fermi surfaces, orbital characters, and interaction parameters, $\mathrm{NaFe}_{0.955} \mathrm{Co}_{0.045}$ As would exhibit an anisotropic gap if the orbital characters or interaction parameters are responsible for the anisotropic behavior here. Therefore, the highly anisotropic gap distribution on the electron pockets of $\mathrm{NaFe}_{0.9825} \mathrm{Co}_{0.0175} \mathrm{As}$ is most likely a direct consequence of the coexisting SDW.

Theories based on the $s^{+-}$paring symmetry have suggested the nodeless and anisotropic gap distribution in the presence of weak SDW [15,16]. Consistently, compared with $\mathrm{NaFeAs}$ [24], much weaker SDW order is present in $\mathrm{NaFe}_{0.9825} \mathrm{Co}_{0.0175} \mathrm{As}$ : The band folding from the SDW order is negligible, and no SDW gap induced by the hybridization with the folded bands is observed here. In a recent theoretical study, it was predicted that even weak SDW order will cause appreciable gap anisotropy [16]. Particularly, that study found that the gap at the tip region of the electron Fermi surface is smaller than that at the flat region, in good agreement with our observation. Futhermore, the observed nodeless SC gap disallows the paring mechanism based on the $s^{++}$pairing symmetry that predicts SC gap nodes in the SDW state $[9,15]$.

The prominent band reconstruction of $\beta$ observed in this paper, with a $32 \mathrm{meV}$ separation between the dispersions along the AFM and FM directions, is smaller than the $46 \mathrm{meV}$ separation observed in NaFeAs [22]. Such a band-reconstruction energy scale is distinct at a specific 


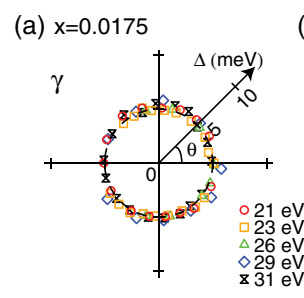

(b) $x=0.0175$

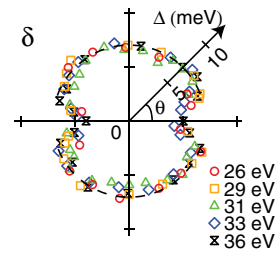

(c) $\mathrm{x}=0.0175$

(d) $x=0.045$

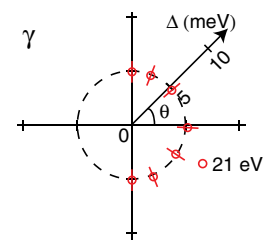

(f)

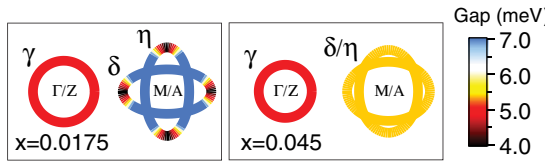

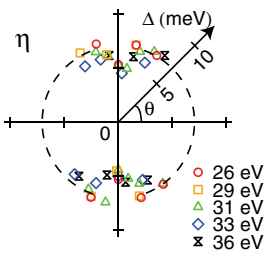

(g)

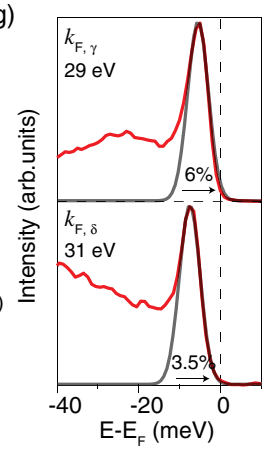

FIG. 4. Polar plots of the SC gap for the (a) $\gamma$, (b) $\delta$, and (c) $\eta$ Fermi surfaces of $\mathrm{NaFe}_{0.9825} \mathrm{Co}_{0.0175} \mathrm{As}$, respectively. The polar angle $\theta$ follows the same definition as in Figs. 2 and 3. The error bar for the gaps is $\pm 1 \mathrm{meV}$ based on the fitting. Polar plots of the SC gap of $\mathrm{NaFe}_{0.955} \mathrm{Co}_{0.045}$ As for the (d) $\gamma$ and (e) $\delta / \eta$ Fermi surfaces, respectively. (f) False-color plots of the gap distribution on the Fermi surfaces of $\mathrm{NaFe}_{0.9825} \mathrm{Co}_{0.0175} \mathrm{As}$ and $\mathrm{NaFe}_{0.955} \mathrm{Co}_{0.045} \mathrm{As}$. (g) The typical spectra at the Fermi crossings of the $\gamma$ and $\delta$ bands taken at $1 \mathrm{~K}$ in BESSY-II. The intensity ratio of the residual spectral weight at $E_{F}$ is referred to the coherence peak height. Two Gaussians with $6 \mathrm{meV}$ full width at half maximum are overlaid.

doping and is correlated with the SDW transition temperature, as observed in $\mathrm{Sr}_{1-x} \mathrm{~K}_{x} \mathrm{Fe}_{2} \mathrm{As}_{2}$ [13]. Therefore, the sharp band dispersion with a single set of bandreconstruction energy scales, plus the resolution-limited width of the superconducting coherent peak [Fig. 4(g)], highlight the homogeneous nature of the electronic state in momentum space. Moreover, although the shielding fraction of the bulk sample is $75 \%$ based on our susceptibility measurements, the ARPES data are taken on a small region $(0.05 \mathrm{~mm} \times 0.2 \mathrm{~mm})$ of the cleaved surface. As shown in Fig. 4(g), the photoemission intensity at $E_{F}$ in the superconducting state is negligible, which suggests the absence of a nonsuperconducting region. In other words, there is no phase separation of superconducting regions and nonsuperconducting SDW regions in the coexisting phase. Our results thus rule out the appearance of macroscopic phase separation and further support the intrinsic coexistence. These results are consistent with a recent STM study on the coexisting phase of $\mathrm{NaFe}_{1-x} \mathrm{Co}_{x}$ As $(x=0.014)$ [21], where the coexistence was found to occur microscopically in an anticorrelated but nonexclusive way between the two orders. Such a nonexclusive coexistence can be understood based on our observation of the indirect competition

between SDW and superconductivity in the electronic structure. Note that the energy scales observed in STM for both the "SDW gap" feature (around $17 \mathrm{meV}$, and it should be a momentum-integrated effect of the band reconstruction) and SC coherence peak (around $5 \mathrm{meV}$ ) are quite independent of space. Those energy scales are further consistent with the single set of SDW and SC energy scales observed in this paper.

Our neutron scattering data on the same sample reveal that static antiferromagnetic long-range order coexists with superconductivity, similar to the static antiferromagnetic order and superconductivity coexisting $\mathrm{BaFe}_{2-x} \mathrm{Ni}_{x} \mathrm{As}_{2}$ samples [14]. The intensity of the SDW diffraction peak decreases upon entering the SC state, suggesting a competition between the two orders [Fig. 1(b)]. The magnitude of the SDW order could be monitored directly from the energy scale of the band reconstruction. However, we did not observe any remarkable change of band reconstruction below $T_{c}$, which suggests that the competition between the two orders does not affect the magnitude of the local SDW order at the fast time scale of photoemission (around $1 \mathrm{fs}$ ). Alternatively, since the itinerant electrons near $E_{F}$ could play an important role in stabilizing the long-range SDW order [37], when the SC gap opens, the coherence of the SDW order could be suppressed. Consequently, the enhanced fluctuation of the local SDW order could be responsible for the observed suppression of the effective (or time-averaged) moment at the quasielastic neutronscattering time scale ( $\gg 1$ ps) [38].

To summarize, we have revealed the detailed electronic structure of the superconductivity and SDW coexisting regime of $\mathrm{NaFe}_{1-x} \mathrm{Co}_{x} \mathrm{As}(x=0.0175)$ and a signature in momentum space for the intrinsic microscopic coexistence. We found that SDW does not cause a noticeable depletion of the states at the Fermi energy, which allows the superconductivity to emerge. Therefore, this result explains why the two orders can coexist in a nonexclusive way. Moreover, we have shown that the anisotropy of the SC gap on the electron pockets is likely a distinct consequence of the coexisting SDW order, while the absence of a gap node puts strong constraints on the pairing symmetry in the theory of iron-based superconductors.

We gratefully acknowledge the helpful discussions with Professor J. P. Hu and Professor A. V. Chubukov, and the experimental support by Dr. D. H. Lu, Dr. M. Hashimoto at SSRL, and Dr. E. Rienks at BESSY-II. This work is supported in part by the National Science Foundation of China and National Basic Research Program of China (973 Program) under Grants No. 2012CB921400, No. 2011CB921802, and No. 2011CBA00112. The single crystal growth efforts and neutron scattering work at the University of Tennessee are supported by the U.S. DOE, BES, through Contract No. DE-FG02-05ER46202. SSRL is operated by the U.S. DOE, BES, Divisions of Chemical Sciences and Material Sciences. 
[1] M. R. Norman, The Challenge of Unconventional Superconductivity, Science 332, 196 (2011).

[2] D. J. Scalapino, A Common Thread: The Pairing Interaction for Unconventional Superconductors, Rev. Mod. Phys. 84, 1383 (2012).

[3] F. Steglich, J. Aarts, C. D. Bredl, W. Lieke, D. Meschede, W. Franz, and H. Schäfer, Superconductivity in the Presence of Strong Pauli Paramagnetism: $\mathrm{CeCu}_{2} \mathrm{Si}_{2}$, Phys. Rev. Lett. 43, 1892 (1979).

[4] P. Limelette, P. Wzietek, S. Florens, A. Georges, T. A. Costi, C. Pasquier, D. Jérome, C. Mézière, and P. Batail, Mott Transition and Transport Crossovers in the Organic Compound $\kappa$-(BEDT-TTF $)_{2} \mathrm{Cu}\left[\mathrm{N}(\mathrm{CN})_{2}\right] \mathrm{Cl}$, Phys. Rev. Lett. 91, 016401 (2003).

[5] Y. Kamihara, T. Watanabe, M. Hirano, and H. Hosono, Iron-Based Layered Superconductor $\mathrm{La}\left[\mathrm{O}_{1-x} \mathrm{~F}_{x}\right] \mathrm{FeAs}$ $(x=0.05-0.12)$ with $T_{c}=26 \mathrm{~K}$, J. Am. Chem. Soc. 130, 3296 (2008).

[6] X. H. Chen, T. Wu, G. Wu, R. H. Liu, H. Chen, and D. F. Fang, Superconductivity at $43 K$ in $\mathrm{SmFeAsO}_{1-x} \mathrm{~F}_{x}$, Nature (London) 453, 761 (2008).

[7] C. de la Cruz, Q. Huang, J. W. Lynn, J. Li, W. Ratcliff, II, J. L. Zarestky, H. A. Mook, G. F. Chen, J. L. Luo, N. L. Wang et al., Magnetic Order Close to Superconductivity in the Iron-Based Layered $\mathrm{LaO}_{1-x} \mathrm{~F}_{x} \mathrm{Fe}$ As Systems, Nature (London) 453, 899 (2008).

[8] D. K. Pratt, W. Tian, A. Kreyssig, J. L. Zarestky, S. Nandi, N. Ni, S. L. Budko, P. C. Canfield, A. I. Goldman, and R. J. McQueeney, Coexistence of Competing Antiferromagnetic and Superconducting Phases in the Underdoped $\mathrm{Ba}\left(\mathrm{Fe}_{0.953} \mathrm{Co}_{0.047}\right)_{2} \mathrm{As}_{2}$ Compound Using $X$-ray and Neutron Scattering Techniques, Phys. Rev. Lett. 103, 087001 (2009).

[9] R. M. Fernandes, D. K. Pratt, W. Tian, J. Zarestky, A. Kreyssig, S. Nandi, M. G. Kim, A. Thaler, N. Ni, P.C. Canfield et al., Unconventional Pairing in the Iron Arsenide Superconductors, Phys. Rev. B 81, 140501(R) (2010).

[10] D. R. Parker, M. J. P. Smith, T. Lancaster, A. J. Steele, I. Franke, P. J. Baker, F. L. Pratt, M. J. Pitcher, S. J. Blundell, and S.J. Clarke, Control of the Competition between a Magnetic Phase and a Superconducting Phase in Cobalt-Doped and Nickel-Doped NaFeAs Using Electron Count, Phys. Rev. Lett. 104, 057007 (2010).

[11] C. Lester, J.-H. Chu, J. G. Analytis, T. G. Perring, I. R. Fisher, and S. M. Hayden, Dispersive Spin Fluctuations in the Nearly Optimally Doped Superconductor $\mathrm{Ba}\left(\mathrm{Fe}_{1-x} \mathrm{Co}_{x}\right)_{2} \mathrm{As}_{2}(x=0.065)$ Phys. Rev. B 81, 064505 (2010).

[12] S. Avci, O. Chmaissem, E. A. Goremychkin, S. Rosenkranz, J.-P. Castellan, D. Y. Chung, I. S. Todorov, J. A. Schlueter, H. Claus, M.G. Kanatzidis et al., Magnetoelastic Coupling in the Phase Diagram of $\mathrm{Ba}_{1-x} \mathrm{~K}_{x} \mathrm{Fe}_{2} \mathrm{As}_{2}$ as Seen via Neutron Diffraction, Phys. Rev. B 83, 172503 (2011).

[13] Y. Zhang, J. Wei, H. W. Ou, J. F. Zhao, B. Zhou, F. Chen, M. Xu, C. He, G. Wu, H. Chen A. Daoud-Aladine, D. Khalyavin, R. Osborn et al., Unusual Doping Dependence of the Electronic Structure and Coexistence of SpinDensity-Wave and Superconductor Phases in Single
Crystalline $\mathrm{Sr}_{1-x} \mathrm{~K}_{x} \mathrm{Fe}_{2} \mathrm{As}_{2}$, Phys. Rev. Lett. 102, 127003 (2009).

[14] H. Q. Luo, R. Zhang, M. Laver, Z. Yamani, M. Wang, X. Y. Lu, M. Y. Wang, Y. C. Chen, S. Li, S. Chang et al., Coexistence and Competition of the Short-Range Incommensurate Antiferromagnetic Order with the Superconducting State of $\mathrm{BaFe}_{2-x} \mathrm{Ni}_{x} \mathrm{As}_{2}$, Phys. Rev. Lett. 108, 247002 (2012).

[15] D. Parker, M. G. Vavilov, A. V. Chubukov, and I. I. Mazin, Coexistence of Superconductivity and a Spin-Density Wave in Pnictide Superconductors: Gap Symmetry and Nodal Lines, Phys. Rev. B 80, 100508(R) (2009).

[16] S. Maiti, R. M. Fernandes, and A. V. Chubukov, Gap Nodes Induced by Coexistence with Antiferromagnetism in Iron-Based Superconductors, Phys. Rev. B 85, 144527 (2012).

[17] J.-Ph. Reid, M. A. Tanatar, X. G. Luo, H. Shakeripour, S. Rene de Cotret, N. Doiron-Leyraud, J. Chang, B. Shen, H.-H. Wen, H. Kim et al., Doping-Induced Vertical Line Nodes in the Superconducting Gap of the Iron Arsenide $\mathrm{Ba}_{1-x} \mathrm{~K}_{x} \mathrm{Fe}_{2} \mathrm{As}_{2}$ from Directional Thermal Conductivity, arXiv:1105.2232 (unpublished).

[18] M.-H. Julien, H. Mayaffre, M. Horvatic, C. Berthier, X. D. Zhang, W. Wu, G.F. Chen, N. L. Wang, and J. L. Luo, Homogeneous vs. Inhomogeneous Coexistence of Magnetic Order and Superconductivity Probed by NMR in Co- and K-Doped Iron Pnictides, Europhys. Lett. 87, 37001 (2009).

[19] Y. Laplace, J. Bobroff, F. Rullier-Albenque, D. Colson, and A. Forget, Atomic Coexistence of Superconductivity and Incommensurate Magnetic Order in the Pnictide $\mathrm{Ba}\left(\mathrm{Fe}_{1-x} \mathrm{Co}_{x}\right)_{2} \mathrm{As}_{2}$, Phys. Rev. B 80, 140501(R) (2009).

[20] X. Zhou, P. Cai, A. Wang, W. Ruan, C. Ye, X. Chen, Y. You, Z.-Y. Weng, and Y. Wang, Evolution from Unconventional Spin Density Wave to Superconductivity and a Pseudogaplike Phase in $\mathrm{NaFe}_{1-x} \mathrm{Co}_{x}$ As, Phys. Rev. Lett. 109, 037002 (2012).

[21] P. Cai, X. Zhou, W. Ruan, A. Wang, X. Chen, D.-H. Lee, and Y. Wang, Visualizing the Microscopic Coexistence of Spin Density Wave and Superconductivity in Underdoped $\mathrm{NaFe}_{1-x} \mathrm{Co}_{x}$ As, arXiv:1208.3842v1 (unpublished).

[22] C. He, Y. Zhang, B. P. Xie, X. F. Wang, L. X. Yang, B. Zhou, F. Chen, M. Arita, K. Shimada, H. Namatame et al., Electronic-Structure-Driven Magnetic and Structure Transitions in Superconducting NaFeAs Single Crystals Measured by Angle-Resolved Photoemission Spectroscopy, Phys. Rev. Lett. 105, 117002 (2010).

[23] C. He, Y. Zhang, X. F. Wang, J. Jiang, F. Chen, L. X. Yang, Z. R. Ye, F. Wu, M. Arita, K. Shimada et al., The Orbital Characters and $\mathrm{kz}$ Dispersions of Bands in Iron-Pnictide NaFeAs, J. Phys. Chem. Solids 72, 479 (2011).

[24] Y. Zhang, C. He, Z. R. Ye, J. Jiang, F. Chen, M. Xu, Q. Q. Ge, B.P. Xie, J. Wei, M. Aeschlimann et al., Symmetry Breaking via Orbital-Dependent Reconstruction of Electronic Structure in Detwinned NaFeAs, Phys. Rev. B 85, 085121 (2012).

[25] M. Yi, D. H. Lu, R. G. Moore, K. Kihou, C.-H. Lee, A. Iyo, H. Eisaki, T. Yoshida, A. Fujimori, Z.-X. Shen, Electronic Reconstruction through the Structural and Magnetic Transitions in Detwinned NaFeAs, New J. Phys. 14, 073019 (2012). 
[26] L. X. Yang, Y. Zhang, H. W. Ou, J. F. Zhao, D. W. Shen, B. Zhou, J. Wei, F. Chen, M. Xu, C. He et al., Electronic Structure and Unusual Exchange Splitting in the SpinDensity-Wave State of the $\mathrm{BaFe}_{2} \mathrm{As}_{2}$ Parent Compound of Iron-Based Superconductors, Phys. Rev. Lett. 102, 107002 (2009).

[27] S. Kasahara, H. J. Shi, K. Hashimoto, S. Tonegawa, Y. Mizukami, T. Shibauchi, K. Sugimoto, T. Fukuda, T. Terashima, Andriy H. Nevidomskyy et al., Electronic Nematicity above the Structural and Superconducting Transition in $\mathrm{BaFe}_{2}\left(\mathrm{As}_{1-x} \mathrm{P}_{x}\right)_{2}$, Nature (London) 486, 382 (2012).

[28] See the Supplemental Material at http://link.aps.org/ supplemental/10.1103/PhysRevX.3.011020 for additional symmetrized EDCs for the comprehensive survey of the superconducting gaps in $\mathrm{NaFe}_{0.9825} \mathrm{Co}_{0.0175} \mathrm{As}$ and $\mathrm{NaFe}_{0.955} \mathrm{Co}_{0.045}$ As.

[29] Z.-H. Liu, P. Richard, K. Nakayama, G.-F. Chen, S. Dong, J.-B. He, D.-M. Wang, T.-L. Xia, K. Umezawa, T. Kawahara et al., Unconventional Superconducting Gap in $\mathrm{NaFe}_{0.95} \mathrm{Co}_{0.05} \mathrm{As}$ Observed by Angle-Resolved Photoemission Spectroscopy, Phys. Rev. B 84, 064519 (2011).

[30] S. V. Borisenko, V. B. Zabolotnyy, A. A. Kordyuk, D. V. Evtushinsky, T. K. Kim, I. V. Morozov, R. Follath, and B. Büchner, One-Sign Order Parameter in Iron Based Superconductor, Symmetry 4, 251 (2012).

[31] K. Umezawa, Y. Li, H. Miao, K. Nakayama, Z.-H. Liu, P. Richard, T. Sato, J. B. He, D.-M. Wang, G. F. Chen et al., Unconventional Anisotropic s-Wave Superconducting Gaps of the LiFeAs Iron-Pnictide Superconductor, Phys. Rev. Lett. 108, 037002 (2012).

[32] K. Okazaki, Y. Ito, Y. Ota, Y. Kotani, T. Shimojima, T. Kiss, S. Watanabe, C. -T. Chen, S. Niitaka, T.
Hanaguri et al., Evidence for a $\cos (\phi)$ Modulation of the Superconducting Energy Gap of Optimally Doped $\mathrm{FeTe}_{0.6} \mathrm{Se}_{0.4}$ Single Crystals Using Laser Angle-Resolved Photoemission Spectroscopy, Phys. Rev. Lett. 109, 237011 (2012).

[33] K. Okazaki, Y. Ota, Y. Kotani, W. Malaeb, Y. Ishida, T. Shimojima, T. Kiss, S. Watanabe, C.-T. Chen, K. Kihou et al., Octet-Line Node Structure of Superconducting Order Parameter in $\mathrm{KFe}_{2} \mathrm{As}_{2}$, Science 337, 1314 (2012).

[34] D. V. Evtushinsky, D. S. Inosov, V. B. Zabolotnyy, A. Koitzsch, M. Knupfer, B. Büchner, M.S. Viazovska, G. L. Sun, V. Hinkov, A. V. Boris et al., Momentum Dependence of the Superconducting Gap in $\mathrm{Ba}_{1-x} \mathrm{~K}_{x} \mathrm{Fe}_{2} \mathrm{As}_{2}$, Phys. Rev. B 79, 054517 (2009)

[35] F. Wang, H. Zhai, D. H. Lee, Nodes in the Gap Function of LaFePO, the Gap Function of the Fe(Se,Te) Systems, and the STM Signature of the \pm \pm Pairing, Phys. Rev. B 81, 184512 (2010).

[36] Y. Zhang, Z. R. Ye, Q. Q. Ge, F. Chen, J. Jiang, M. Xu, B.P. Xie, and D.L. Feng, Nodal SuperconductingGap Structure in Ferropnictide Superconductor $\mathrm{BaFe}_{2}\left(\mathrm{As}_{0.7} \mathrm{P}_{0.3}\right)_{2}$, Nat. Phys. 8, 371 (2012).

[37] M.D. Johannes and I. I. Mazin, Microscopic Origin of Magnetism and Magnetic Interactions in Ferropnictides, Phys. Rev. B 79, 220510(R) (2009).

[38] P. Vilmercati, A. Fedorov, F. Bondino, F. Offi, G. Panaccione, P. Lacovig, L. Simonelli, M. A. McGuire, A.S.M. Sefat, D. Mandrus et al., Itinerant Electrons, Local Moments, and Magnetic Correlations in the Pnictide Superconductors $\mathrm{CeFeAsO}_{1-x} \mathrm{~F}_{x}$ and $\mathrm{Sr}\left(\mathrm{Fe}_{1-x} \mathrm{Co}_{x}\right)_{2} \mathrm{As}_{2}$, Phys. Rev. B 85, 220503(R) (2012). 